\title{
Avaliação da qualidade de vida em diabéticos e/ou hipertensos usuários de um núcleo de apoio à saúde da família
}

\author{
Quality of life assessment in diabetic and / or hypertensive \\ users of a support center health of family
}

RESumo Este estudo teve como objetivo avaliar a qualidade de vida em diabéticos e/ou hipertensos acompanhados pelo Núcleo de Apoio a Saúde da Família de Canindé, Ceará. Trata-se de um estudo de corte transversal com análise qualiquantitativa. A amostra foi composta por 45 diabéticos e/ou hipertensos (24 ativos e 21 não ativos), de ambos os sexos, com idade média de 52,23 $( \pm 11,25)$ anos. A qualidade de vida foi avaliada por meio de entrevista e a aplicação do questionário Whoqol Bref. Os dados qualitativos foram analisados de forma descritiva e interpretativa pela análise do conteúdo. Os dados quantitativos foram analisados por intermédio do teste de Mann-Whitney no programa SPSS ${ }^{\circ}$ for Windows 10, expressos como média e desvio padrão da média. O nível de significância estabelecido foi de $\mathrm{p}<0,05$. Os resultados apontaram a melhora da qualidade de vida dos diabéticos e hipertensos ativos em comparação aos não ativos em todos os domínios avaliados, independentemente da doença ou de gênero. Esses resultados foram fortalecidos pelos depoimentos dos usuários ao reconhecer a redução de suas limitações físicas, o fortalecimento da autoestima e motivação, as relações interpessoais existentes e o ambiente saudável e acessível. Este estudo mostrou a importância da prática de exercícios físicos na melhora da qualidade de vida de seus usuários e da inserção do profissional de Educação Física dentro da equipe multiprofissional do Núcleo de Apoio à Saúde da Família, na perspectiva da integralidade do cuidado em saúde.

Palavras-chave: Qualidade de vida. Hipertensão. Diabetes Mellitus. SAÚDE DA FAMÍlIA.

Marcella Mayara Sousa Silva ${ }^{\mathrm{I}}$ Francisco Laerton Teixeira GUERRA $^{I}$

MARINA LiRa Batista ${ }^{1}$

LIDALVANe SiLVA Lopes ${ }^{\text {II }}$

Samara Moura BarReto de ABreU ${ }^{I}$

Nilson Vieira Pinto

'Instituto Federal de Educação, Ciência e Tecnologia do Ceará (IFCE) IISecretaria Municipal de Saúde de Canindé/CE
Abstract This study aimed to evaluate the quality of life of diabetics and/or hypertensive patients under the health care assistance of Support Center Health of Family at Canindé-Ceará. This is a cross-sectional study with qualitative and quantitative analysis. The sample comprehended 45 diabetics and/or hypertension patients ( 24 active and 21 no active), of both sexes, with an average of 52.23 \pm 11.25 years of age. Quality of life was assessed through an interview and the use of the Whoqol Bref questionnaire. Qualitative data were analysed in a descriptive and interpretative way by the analysis of the content. Quantitative data were analysed using Mann-Whitney test by SPSS program for Windows 10, expressed as mean and standard deviation of the mean. The significance level was $\mathrm{p}<0.05$, previously established. The results showed the improvement of the quality of life of the active diabetics and hypertensive individuals in comparison to the no active in all areas evaluated, regardless of gender or disease. These results were strengthened through the testimonials of users by recognizing the reduction of their physical limitations, the strengthening of self-esteem and motivation, interpersonal relationships, and the health environment and its accessibility. This study showed the importance of the practice of the physical exercise on the improvement of the quality of life of its users and the professional insertion of physical education within the multidisciplinary team of the Support Center Health of Family. Keywords: Quality of life. Hypertension. Diabetes Mellitus. Public HEALTH. 


\section{INTRODUÇÃO}

$\mathrm{O}$ diabetes mellitus (DM) e a hipertensão arterial sistêmica (HAS) são doenças crônicas, recorrentes, de origem múltipla que afetam diretamente o metabolismo do indivíduo, cujo controle e a diminuição dos fatores de risco dependem de mudanças no estilo de vida, dieta, ingestão de medicamentos e prática de atividade física regular. Essas mudanças refletem de forma positiva na qualidade de vida de diabéticos e hipertensos, quando há uma orientação adequada, seguida da adesão ao tratamento. ${ }^{1}$

A Qualidade de Vida (QV), por sua vez, pode ser representada pelas inter-relações existentes entre o meio ambiente, o social, $o$ psicológico e seus aspectos físicos. ${ }^{2}$ Refere-se à forma como o indivíduo se percebe enquanto sujeito de sua história, em seu contexto cultural, e sistema de valores no qual se insere em relação aos seus objetivos, expectativas, padrões e preocupações, ${ }^{3}$ e está atualmente relacionada a parâmetros que possam ir além do controle da doença, diminuição da mortalidade ou aumento da expectativa de vida. ${ }^{4}$

Dentro das estratégias de promoção da saúde promovidas pelo Ministério da Saúde que podem contribuir para a melhora da QV de diabéticos e hipertensos, encontram-se os Núcleos de Apoio à Saúde da Família (NASFs). Esses núcleos devem ser compostos por uma equipe multidisciplinar capaz de interferir diretamente em todas as dimensões qualitativas e quantitativas dessa relação $0^{5}$ na interface da clínica ampliada e apoio matricial.

Inserido na equipe multidisciplinar encontra-se o profissional de Educação Fí- sica, responsável pelo desenvolvimento das atividades físicas/práticas corporais, contribuindo para a ampliação de uma população fisicamente ativa e, por conseguinte, na promoção da saúde e da qualidade de vida desses usuários. ${ }^{5}$

Alguns estudos ${ }^{1,7}$ têm avaliado a QV de diabéticos e hipertensos acompanhados pela Equipe de Saúde da Família, embora até o momento pouco se tenha correlacionado essa interface com a atividade física. Sendo assim, o presente estudo veio avaliar sob uma ótica dimensional a QV em diabéticos e/ou hipertensos, ativos e não ativos, acompanhados pelo NASF do município de Canindé.

\section{Métodos}

\section{Sujeitos da pesquisa}

Trata-se de uma amostragem não probabilística e intencional composta por 45 diabéticos e/ou hipertensos (24 ativos, sendo 22 do gênero feminino e 2 do masculino e 21 não ativos, sendo 14 do gênero feminino e 7 do masculino) assistidos pelo Núcleo de Apoio à Saúde da Família (NASF) da zona urbana do município de Canindé-Ceará, com idade média de 52,23 $\pm 11,25$.

Foram considerados ativos aqueles que praticavam atividade física regular a um tempo mínimo de três meses. O NASF orientava um programa de exercícios físicos predominantemente aeróbicos realizados duas vezes por semana sob a orientação do profissional de Educação Física. Entre os critérios de inclusão, além dos já citados, atentou-se para a assiduidade de no mínimo $75 \%$ de frequência no programa de atividade física. Foram excluídos do estudo os in- 
divíduos em acompanhamento temporário (menos de três meses de participação no programa de atividade física); portadores de transtornos mentais e os que não preencheram os critérios de inclusão mencionados.

Todos os participantes investigados inicialmente foram informados sobre os objetivos e delineamentos da pesquisa e em seguida assinaram o Termo de Consentimento Livre e Esclarecido (TCLE). Este estudo seguiu-se dentro das normas que regulamentam a pesquisa em seres humanos, do Conselho Nacional de Saúde - Ministério da Saúde, Resolução No. 510/2016. Este estudo encontra-se aprovado pelo CEP/IFCE sob o Parecer nº. 432.503/2013.

\section{Procedimentos de coleta de dados}

A qualidade de vida dos usuários foi avaliada quantitativamente por meio da aplicação do questionário Whoqol Bref on-line e apreciada qualitativamente por entrevista, realizada no período de abril a julho de 2016.

O questionário Whoqol Bref on-line é um instrumento constituído de 26 perguntas que avaliaram conceitualmente os domínios: físicos, psicológicos, relações sociais e de meio ambiente, na percepção de saúde desses usuários. Esse instrumento foi escolhido por ser uma versão mais simplificada do WHOQOL-100, contendo as perguntas mais relevantes para este estudo e por sua validade científica.

No domínio físico, são abordados as necessidades e sentimentos como: dor, tratamento médico, disposição em geral, mobilidade e repouso. No domínio psicológico, discutem-se temas como: aproveitar a vida, sentido de viver, concentração, aceitação da aparência física, satisfação pessoal e sentimentos negativos. No domínio relações sociais, a satisfação dos entrevistados quanto a: relações pessoais, sexual e amizades, e no domínio meio ambiente, consideram-se situações como: segurança, ambiente saudável, finanças, informatividade, lazer, condições de moradia, acesso aos serviços de saúde e meio de transporte.

Os resultados dos domínios apresentam valores entre zero e cem, sendo piores os mais próximos de zero e melhores os mais próximos de cem. Dessa forma, um indivíduo que mostre valor igual a 50 para determinado domínio pode ser considerado mediano para esse domínio.

A entrevista foi composta por duas perguntas direcionadas somente ao grupo amostral: 1. Como o (a) senhor (a) se sentia antes de fazer parte do NASF? 2. Como o (a) senhor (a) se sente hoje, fazendo parte desse grupo? Os entrevistados tiveram liberdade em tempo e expressão para respondê-las. As respostas foram gravadas para posterior transcrição, análise interpretativa e categorização.

\section{Análise dos dados}

Os dados quantitativos foram tabulados inicialmente em um banco de dados com base em uma planilha em Excel (windows office 2016), em seguida analisados por meio do teste de Mann-Whitney no programa SPSS for Windows 10 e expressos como média e desvio padrão da média. O nível de significância estabelecido foi de $p<0,05$. Os dados qualitativos tiveram uma apreciação descritiva e interpretativa pela 
análise do conteúdo seguindo os pressupostos de Pope e Mays. ${ }^{8}$

\section{RESUltados e discussão}

A QV dos diabéticos e/ou hipertensos (ativos e não ativos) foi avaliada quantitativamente por intermédio da aplicação do questionário Whoqol Bref e apreendida qualitativamente por meio de entrevistas aos usuários ativos, como representação e significado do programa de atividade física realizado pelo NASF Canindé. Dessa forma, foram analisados 45 questionários e 16 entrevistas, sendo 24 usuários ativos e 21 não ativos.

A avaliação do Whoqol Bref nos diabéticos e/ou hipertensos ativos mostrou-se estatisticamente diferente dos não ativos em todos os domínios avaliados (Tabela 1).

Tabela 1. Média e desvio-padrão dos escores dos domínios de QV (Whoqol Bref)

\begin{tabular}{lccccc}
\hline & \multicolumn{2}{c}{$\begin{array}{c}\text { ATIVOS } \\
(n=24)\end{array}$} & \multicolumn{2}{c}{$\begin{array}{c}\text { NÃO ATIVOS } \\
(n=21)\end{array}$} & \\
\cline { 2 - 5 } \multicolumn{1}{c}{$\begin{array}{c}\text { Domínios da Qualidade de } \\
\text { Vida (WHOQOL/bref) }\end{array}$} & Média & DP & Média & DP & $p$ \\
\hline Físico & 73,6 & 11,3 & 46,2 & 11,1 & 0,0003 \\
Psicológico & 75 & 13,4 & 49,3 & 9,7 & 0,0173 \\
Relaçóes Sociais & 77,4 & 13,3 & 40,3 & 12,7 & 0,0047 \\
Meio Ambiente & 74,4 & 11,2 & 47,1 & 13,4 & 0,0052 \\
\hline
\end{tabular}

Nota: ${ }^{*}(\mathrm{p}<.05), D P=$ Desvio Padrão.

Fonte: Os autores.

No domínio físico, os usuários ativos apresentaram escore médio de $73,6 \pm 11,3$, enquanto os não ativos 46,2 $\pm 11,1$ $(\mathrm{p}=0,0003)$. Tal constatação é significada a partir das narrativas dos sujeitos em relação à experiência vivida junto ao programa de atividade física desenvolvido pelo NASF de Canindé:

\footnotetext{
Não me sentia bem, vivia com a pressão alta e não sabia, aí a agente de saúde me indicou pra eu ir ao posto, lá na clínica, ai comecei a me tratar, faz 20 anos que me trato e agora nessas físicas eu me sinto muito bem, minha pressão toda vida na consulta dá boa 12,13, 14 é o número da minha pressão, graças a Deus. (Usuário ativo 1.)

Sentia o corpo pesado, sem movimento, dor nas costas, nas pernas, depois que comecei a vim pra cá não sinto mais nada, eu faço tudo graças a
}

Deus, rodo por todo canto, vou pra rua, trabalho todo dia. (Usuário ativo 2.)

É possível perceber evidências sobre o processo da melhoria da QV, cuja inferência é feita sobre a minimização da pressão arterial e associação de dores corporais; a mobilidade física e processos de relaxamento, otimizando as atividades diárias pela relativa autonomia. A respeito dessa realidade significada, Bündchen et al., ${ }^{9}$ identificaram que a prática de exercícios físicos em comparação ao tratamento farmacológico convencional possibilitou idêntico controle da pressão arterial e melhor percepção de qualidade de vida. Santos ${ }^{10}$ associou a prática de exercícios físicos a uma melhor aptidão físi- 
ca e qualidade de vida em mulheres idosas e diabéticas.

No domínio psicológico, os usuários ativos obtiveram escore médio de $75 \pm 13$, 4 , enquanto os não ativos 49,3 $\pm 9,7(\mathrm{p}=0,0173)$. Diante dos excertos das falas dos sujeitos, identifica-se a racionalidade psicológica aferida positivamente, pela melhoria da imagem corporal e da apreensão da felicidade produzida pela participação no programa:

[...] Quando comecei a fazer parte do grupo melhorou muito, renovou minha vida, todo mundo acha eu é nova, até o doutor acha eu nova, por que eu digo que tenho 76 anos e todo mundo diz que não tem quem diga e as enfermeiras também e me sinto bem graças a Deus. (Usuário ativo 3.) Antes de fazer parte do grupo eu era uma pessoa e agora me tornei outra, mais alegre, mais feliz e melhor de saúde, tudo de bom, o grupo para mim é uma maravilha. (Usuário ativo 4.)

Nessa alusão, torna-se importante fazer uma leitura sobre a QV, direcionando significados à busca da felicidade e realização de potenciais pessoais e coletivos. ${ }^{11}$

No domínio relações sociais, os usuários ativos tiveram escore médio de $77,4 \pm 13,3$, enquanto os não ativos 40,3 $\pm 12,7$ $(\mathrm{p}=0,0047)$. Esse domínio também, pode ser identificado nas narrativas dos sujeitos:

Eu me sentia muito sozinha, aqui a gente tem a turma de amigos, a gente faz as amizades, às vezes a gente tá em casa tão estressada, a gente vem pra cá é tão bom. (Usuário ativo 5.)

Entoa-se o processo de convivialidade e urbanidade pelas inter-relações produzidas no espaço grupal, como condição geradora da QV. Nesse contexto, Boff ${ }^{12}$ alude que o cuidado em saúde, como dimensão da QV, se configura como atitude de preocupação, de responsabilidade e de envolvimento afetivo com o outro.

No domínio meio ambiente, os usuários ativos alcançaram escore médio de $74,4 \pm 11,2$, enquanto os não ativos $47,1 \pm 13,4$ $(\mathrm{p}=0,0052)$. De forma significativa, a relação positiva sobre o domínio meio ambiente foi aferida pelos sujeitos, na razão da sustentabilidade e economicidade financeira, assim como, na acessibilidade aos serviços de saúde como processo de cuidado longitudinal:

Mudou muito por que eu tô com mais saúde e tem essas ajudas desses exames né, já é outra ajuda para gente, por que as vezes nem que você queira fazer, você num pode e com esse exame que a gente faz aqui é uma ajuda muito boa para gente, eu me sinto muito feliz e agradeço demais. (Usuário ativo 6.)

Antes eu nem sabia o que eu sentia, no dia que eu vim para cá, minha pressão deu muito alta 18 por não sei por quanto, aí as meninas aqui ajeitaram, eu era assim sem me cuidar, nunca liguei muito para esse lado, mas agora faz quatro aninhos que eu tô me cuidando aqui, aqui bem acompanhada com a menina (se referindo a profissional de educação física do NASF) e com vocês, sempre vem, de dois e dois meses vem uma parte de jovens nos ajudar, aí agora eu tô ótima, antes não me cuidava eu ia uma consulta uma vez na vida, mas nem isso eu ligava para ir, notei que melhorei muito, faço mais uma caminhadinha perto ali de casa, caminhadinha 5 horas da manhã, ela mesmo diz que a gente pode fazer fora daqui, ela orienta. (Usuário ativo 7.)

[...] Um mês sim e outro não, tenho minha consulta, para receber meus remédios e quando não tem eu compro e sei que graças a Deus eu vou levando. (Usuário ativo 8.)

Conforme anuncia Minayo, ${ }^{13}$ vislumbra-se como determinantes de saúde as condições de alimentação, habitação, educação, 
renda, meio ambiente, trabalho, emprego, lazer, liberdade.

Em uma perspectiva de análise dos domínios de QV por gênero, pôde-se identificar que os usuários ativos se mostraram diferentes estatisticamente dos não ativos, em todos os domínios avaliados, independentemente do gênero, entretanto indiferentes quanto ao gênero, quando comparados entre si (Tabela 2).

Em detalhe, no domínio físico, os usuários ativos do gênero feminino apresentaram escore médio de $72,5 \pm 7,6$, mostrando-se estatisticamente diferente $(\mathrm{p}<0,05)$ do grupo não ativo $(44,6 \pm 10,4)$, entretanto não exibindo distinção em relação aos pacientes ativos do gênero masculino $(80,7 \pm 4,2)$.

No domínio psicológico, os usuários ativos do gênero feminino obtiveram escore médio de $74,2 \pm 5,7$, mostrando-se estatisticamente diferente $(\mathrm{p}<0,05)$ do grupo não ativo $(47,7 \pm 11,3)$, contudo, não se diferenciando em relação aos pacientes ativos do gênero masculino $(83,3 \pm 5,1)$.

No domínio relações sociais, os pacientes ativos do gênero feminino tiveram escore médio de $78,4 \pm 12,3$, mostrando-se estatisticamente diferente $(\mathrm{p}<0,05)$ do grupo não ativo $(41,1 \pm 5,7)$, porém, sem distinção em relação aos pacientes ativos do gênero masculino $(66,6 \pm 13,5)$.

No domínio meio ambiente, os pacientes ativos do gênero feminino alcançaram escore médio de $74,3 \pm 11,3$, mostrando-se estatisticamente diferentes $(\mathrm{p}<0,05)$ do grupo não ativo $(45,7 \pm 14,8)$, todavia, indiferente em relação aos pacientes ativos do gênero masculino $(69,1 \pm 9,2)$. Vale ressaltar, por sua vez que, assim como, no gênero feminino, os pacientes ativos do gênero masculino, se diferem estatisticamente dos não ativos, mas não do gênero feminino de mesmo nível de atividade física em todos os domínios avaliados.

Em continuidade, destaca-se que mesmo em uma análise comparativa por caracterização patológica entre os grupos de pacientes diabéticos, hipertensos e diabéticos-hipertensos, pode-se verificar diferenças significativas entre os ativos e os não ativos, em todos os domínios avaliados (Tabela 3).

No domínio físico, os pacientes diabéticos ativos apresentaram escore médio de $71,4 \pm 11,4$, diferente estatisticamente $(\mathrm{p}=0,0001)$ dos diabéticos não ativos $(43,6 \pm 11,7)$. De forma semelhante, os hiper-

Tabela 2. Média e desvio-padrão dos escores dos domínios de QV (Whoqol Bref) analisados por gênero.

\begin{tabular}{lcccccccc}
\hline & \multicolumn{3}{c}{ ATIVOS } & \multicolumn{3}{c}{ NÃO ATIVOS } \\
$\begin{array}{l}\text { Domínios da Qualidade de } \\
\text { Vida (WHOQOL/bref) }\end{array}$ & \multicolumn{2}{c}{$\begin{array}{c}\text { Feminino } \\
(n=22)\end{array}$} & $\begin{array}{c}\text { Masculino } \\
(n=2)\end{array}$ & \multicolumn{2}{c}{$\begin{array}{c}\text { Feminino } \\
(n=14)\end{array}$} & $\begin{array}{c}\text { Masculino } \\
(n=7)\end{array}$ \\
\cline { 2 - 10 } & Média & $D P$ & Média & $D P$ & Média & $D P$ & Média & $D P$ \\
\hline Físico & $72,5^{*}$ & 7,6 & $80,7^{*}$ & 4,2 & 44,6 & 10,4 & 43,4 & 13,9 \\
Psicológico & $74,2^{*}$ & 5,7 & $83,3^{*}$ & 5,1 & 47,7 & 11,3 & 42,5 & 11,7 \\
Relações Sociais & $78,4^{*}$ & 12,3 & $66,6^{*}$ & 13,5 & 41,1 & 5,7 & 47,6 & 5,4 \\
Meio Ambiente & $74,3^{*}$ & 11,3 & $69,1^{*}$ & 9,2 & 45,7 & 14,8 & 44,0 & 12,5 \\
\hline
\end{tabular}

Nota: ${ }^{*}$ comparação entre ativos e não ativos do mesmo gênero, $D P=$ Desvio Padrão.

Fonte: Os autores. 
tensos ativos $(70,9 \pm 9,2)$ se mostraram distintos dos hipertensos não ativos $(47,5 \pm 7,4)$, assim como, houve diferenças entre os usuários ativos $(75 \pm 9,9)$ e não ativos $(40,7 \pm 16,6)$ que tinham as duas patologias.

No domínio psicológico, os diabéticos ativos obtiveram escore médio de 79,8 48,6 , diferente estatisticamente $(\mathrm{p}=0,0005)$ dos diabéticos não ativos $(44,3 \pm 14,2)$. De maneira igual, os hipertensos ativos $(76,1 \pm 5,2)$ se apresentaram diferentes dos hipertensos não ativos $(46,3 \pm 7,1)$, assim como, ocorreram diferenças entre os usuários ativos $(73,6 \pm 7,6)$ e não ativos $(51,3 \pm 7,7)$ que tinham as duas enfermidades.

No domínio relações sociais, os diabéticos ativos alcançaram escore médio de $79,3 \pm 7,2$, diferente estatisticamen- te $(\mathrm{p}=0,0003)$ dos diabéticos não ativos $(40,2 \pm 12,8)$. Semelhantemente, os hipertensos ativos $(80,6 \pm 10,1)$ se mostraram diferentes dos hipertensos não ativos $(44,2 \pm 8,6)$, assim como, houve diferenças entre os usuários ativos $(77 \pm 8,5)$ e não ativos $(41,4 \pm 9,4)$ diabéticos e hipertensos.

No domínio meio ambiente, os diabéticos ativos tiveram escore médio de $72,9 \pm 11,2$, diferente estatisticamente $(\mathrm{p}=0,0178)$ dos diabéticos não ativos $(46,4 \pm 9,7)$. Os hipertensos ativos $(77,3 \pm 8,3)$ se apresentaram diferentes dos hipertensos não ativos $(53,1 \pm 7,4)$, assim como, ocorreram diferenças entre os usuários ativos $(76,5 \pm 7,4)$ e não ativos $(41,9 \pm 16,8)$ diabéticos e hipertensos.

Tabela 3. Média e desvio-padrão dos escores dos domínios de QV (Whoqol Bref on-line) em relação à caracterização da doença.

\begin{tabular}{|c|c|c|c|c|c|c|}
\hline & & \multicolumn{5}{|c|}{ Domínios da Qualidade de Vida (WHOQOL/bref) } \\
\hline & & & Físico & Psicológico & $\begin{array}{l}\text { Relações } \\
\text { Sociais }\end{array}$ & $\begin{array}{c}\text { Meio } \\
\text { Ambiente }\end{array}$ \\
\hline \multirow{6}{*}{ 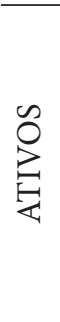 } & \multirow[t]{2}{*}{ Diabéticos } & Média & 71,4 & 79,8 & 79,3 & 72,9 \\
\hline & & $D P$ & 11,4 & 8,6 & 7,2 & 11,2 \\
\hline & \multirow[t]{2}{*}{ Hipertensos } & Média & 70,9 & 76,1 & 80,6 & 77,3 \\
\hline & & $D P$ & 9,2 & 5,2 & 10,1 & 8,3 \\
\hline & \multirow{2}{*}{$\begin{array}{l}\text { Diabéticos e } \\
\text { Hipertensos }\end{array}$} & Média & 75 & 73,6 & 77 & 76,5 \\
\hline & & $D P$ & 9,9 & 7,6 & 8,5 & 7,4 \\
\hline \multirow{7}{*}{ 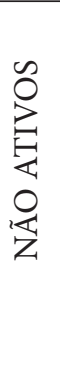 } & \multirow[t]{2}{*}{ Diabéticos } & Média & 43,6 & 44,3 & 40,2 & 46,4 \\
\hline & & $D P$ & 11,7 & 14,2 & 12,8 & 9,7 \\
\hline & \multirow[t]{2}{*}{ Hipertensos } & Média & 47,5 & 46,3 & 44,2 & 53,1 \\
\hline & & $D P$ & 7,4 & 7,1 & 8,6 & 7,4 \\
\hline & \multirow{3}{*}{$\begin{array}{l}\text { Diabéticose } \\
\text { Hipertensos }\end{array}$} & Média & 40,7 & 51,3 & 41,4 & 41,9 \\
\hline & & $D P$ & 16,6 & 7,7 & 9,4 & 16,8 \\
\hline & & $P$ & 0,0001 & 0,005 & 0,0003 & 0,0178 \\
\hline
\end{tabular}

Nota: ${ }^{\star}(\mathrm{p}<.05), D P=$ Desvio Padrão.

Fonte: Os autores. 
Mota et al. ${ }^{14}$ compararam a qualidade de vida entre participantes e não participantes de programas formais de atividade física, identificando a melhora na qualidade de vida relacionada à saúde por meio da participação em programas de atividade física. Interdonato e Greguol ${ }^{15}$ investigaram a qualidade de vida entre ativos e não ativos, tendo os ativos apresentado melhor percepção de qualidade de vida, e, assim como no nosso estudo, independentemente do gênero. Silva et al. ${ }^{16}$ avaliaram as associações da prática de atividade física na qualidade de vida dos indivíduos e apontaram que quanto mais ativo, melhor a qualidade de vida do indivíduo. Recentemente, Da Costa et al. ${ }^{17}$ compararam a qualidade de vida, equilíbrio e força muscular em idosos da cidade de Goiás, praticantes e não praticantes de atividade física e identificaram que os ativos exibiram melhor qualidade de vida, equilíbrio e força muscular que os não ativos.

Não obstante, resultado semelhante tem sido observado em pacientes diabéticos e/ ou hipertensos participantes de programa de atividade física. Caran e Santos ${ }^{18}$ caracterizaram um grupo de idosas diabéticas que ampliaram sua qualidade de vida por meio da prática de exercícios físicos regulares.

Fortalecendo ainda mais esses resultados em um olhar junto aos serviços de saúde, Ferreira; Diettrich; Pedro ${ }^{19}$ investigaram a influência da atividade física sobre a qualidade de vida de participantes e não participantes de um Programa de Atividade Física, atendidos nas Unidades Básicas de Saúde da Família, no município de Campo Grande-MS, e apontaram tal prática como contribuinte na melhora da qualidade de vida em programas de promoção da saúde. Pereira;
Borges; Amadei ${ }^{7}$ verificaram a qualidade de vida de portadores de hipertensão arterial em tratamento medicamentoso, atendidos na rede pública de saúde do município de Atalaia-Paraná, e perceberam que a mudança dos hábitos de vida, advindos de uma assistência organizada e direcional, contribui para a melhora da qualidade de vida.

Diante desses achados, ficou evidenciado a melhora da qualidade de vida nos diabéticos e/ou hipertensos ativos acompanhados pelo NASF de Canindé. Tal reconhecimento fortalece as ações de planejamento desenvolvidas pelo profissional de Educação Física inserido ativamente na equipe de atenção básica à saúde e merecem ser amplamente divulgadas, sobretudo, na Política de Atenção à Saúde, de modo evidente na contextualidade na esfera municipal, junto à equipe multidisciplinar, aos gestores da $5^{\mathrm{a}}$ Coordenadoria Regional de Saúde e a toda a comunidade canindeense, no intuito de se ampliar a participação e adesão dos usuários nesse serviço de apoio à saúde.

Entre as limitações deste estudo, destacam-se o exíguo material científico-bibliográfico que aborde a qualidade de vida em diabéticos e hipertensos, especialmente em pacientes ativos e participantes de programas de atividade física vinculados a unidades básicas de saúde. Em adição, o quantitativo de usuários avaliados, que embora significativo, ainda ínfero, se comparado ao número total de diabéticos e hipertensos existentes em Canindé-Ceará.

\section{CONSIDERAÇões Finais}

A avaliação da qualidade de vida dos diabéticos e/ou hipertensos ativos assisti- 
dos pelo NASF em Canindé mostrou-se estatisticamente diferente dos não ativos em todos os domínios avaliados nesta amostra, independentemente do gênero. Entretanto, indiferentes quanto ao gênero quando comparados entre si. Mesmo em uma análise comparativa entre os grupos de diabéticos, hipertensos e diabéticos-hipertensos, pôde-se verificar, diferenças significativas entre os ativos e os não ativos em todos os domínios avaliados.

Estes resultados foram fortalecidos e reconhecidos por meio dos depoimentos dos usuários, o que enfatizou a importância desses usuários em participar de programas de atividade física. $\mathrm{O}$ efeito da atividade física refletida na qualidade de vida dos diabéticos e/ou hipertensos, no aspecto físico, diminuiu as limitações desses usuários, tornando-os mais independentes e os auxiliando no autocuidado; no aspecto psicológico tornando-os mais alegres e motivados na boa convivência com sua patologia; no aspecto social por intermédio das inter-relações com outras pessoas, trocas de experiências, conversas, amizades e no domínio meio ambiente referindo-se ao ambiente saudável, ao acesso às informações e com isso a maior procura aos serviços de saúde.

Diante disso, ressalta-se a importância da prática de exercícios físicos na melhora da qualidade de vida de seus praticantes e enobrece o profissional de Educação Física como um importante personagem dessa ação, configurando sua importância dentro da equipe multiprofissional do NASF, na perspectiva da integralidade do cuidado em saúde.

Espera-se a partir destes resultados ampliar a reflexão sobre a qualidade de vida proporcionada aos usuários do NASF, promovendo amplas/novas estratégias de educação em saúde, visando a adoção de um estilo de vida mais saudável e promoção do autocuidado, resultando na melhora da qualidade de vida dos mesmos para além da atividade física.

\section{REFERÊNCIAS}

1. Miranzi SDSC, Ferreira FS, Iwamoto HH, Pereira GDA, Miranzi MAS. Qualidade de vida de indivíduos com diabetes mellitus e hipertensão acompanhados por uma equipe de saúde da família. Texto Contexto Enfermagem 2008; 17 (4): 672.

2. Zanetti ML, Biagg MV, Santos MAD, Péres DS, Teixeira CRDS. O cuidado à pessoa diabética e as repercussões na família. Revista Brasileira de Enfermagem 2008; 61 (2):186-192.

3. Almeida MAB, Gutierrez, GL, Marques R. Qualidade de vida: definição, conceitos e interfaces com outras áreas, de pesquisa, Escola de Artes, Ciências e Humanidades - EACH/USP - São Paulo, 142 p, 2012.

4. Pereira ÉF, Teixeira CS, Santos A. Qualidade de vida: abordagens, conceitos e avaliação. Revista Brasileira Educação Física Esporte 2012; 26 (2): 241-250.

5. Rodrigues JD, Ferreira D., Silva P., Caminha I., de Farias Junior JC. Inserção e atuação do profissional de educação física na atenção básica à saúde: revisão sistemática. Revista Brasileira de Atividade Física \& Saúde 2013; 18 (1): 05-15.

6. Brasil Ministério da Saúde. Diretrizes do NASF: Núcleo de Apoio a Saúde da Família / Ministério da Saúde. Secretaria de Atenção à Saúde, Departamento de Atenção Básica. (Série A. Normas e Manuais Técnicos) (Caderno de Atenção Básica, n. 27). Brasília: Ministério da Saúde, 152 p. 2010. 
7. Pereira CL, Borges MS, Amadei JL. Qualidade de vida de hipertensos usuários da rede pública de saúde. Revista Saúde e Pesquisa 2013; 6 (2): 295-303.

8. Pope C., Mays N. Pesquisa qualitativa na atenção à saúde, 3. ed. Porto Alegre: Artmed; 2009.

9. Bundchen DC, Schenkel IDC, Santos RZD, Carvalho TD. Exercício físico controla pressão arterial e melhora qualidade de vida. Rev Bras Med Esporte 2013; 19 (2): 91-5.

10. Santos APA. Associação entre atividade física, aptidão física e qualidade de vida em pessoas idosas com Diabetes Mellitus tipo 2 [Dissertação de Mestrado em Exercício e Saúde]. Évora: Universidade de Évora. Escola de Ciências e Tecnologia. Departamento de desporto e saúde, 2015.

11. Marcondes, WB. A convergência de referências na promoção da saúde. Saúde e Sociedade 2004; 13 (1): 5-13.

12. Boff L. Saber cuidar: ética do humano - compaixão pela terra. Petrópolis: Vozes, 1999.

13. Minayo MCS. Enfoque ecossistêmico de saúde e qualidade de vida. In: Minayo, MCS. \& Miranda AC. Saúde e ambiente sustentável: estreitando nós. Rio de Janeiro: Editora FIOCRUZ; 2002.

14. Mota J, Ribeiro JL, Carvalho J, MATOS MG. Atividade física e qualidade de vida associada à saúde em idosos participantes e não participantes em programas regulares de atividade física. Revista brasileira de educação física e esporte, 2006; 20 (3): 219-225.

15. Interdonato GC, Greguol M. Qualidade de Vida Percebida por Indivíduos Fisicamente Ativos e Não ativos. R. bras. Ci. e Mov 2010; 18 (1): 61-67.

16. Silva RS, Silva I., Silva RA, Souza L., Tomasi E. Atividade física e qualidade de vida. Ciência \& Saúde Coletiva 2010; 15 (1): 115-120.

17. Da Costa LDSV, de Sousa NM, Alves AG, de Brito FAV, Araújo RF, Nogueira MS. Análise comparativa da qualidade de vida, equilíbrio e força muscular em idosos praticantes de exercício físico e não ativos. Revista Eletrônica Faculdade Montes Belos 2015; 8 (3): 63-179.

18. Caran DG, Dos Santos KP. Exercício físico regular e qualidade de vida em mulheres com Diabetes Mellitus tipo 2. RBPFEX-Revista Brasileira de Prescrição e Fisiologia do Exercício 2011; 5 (28): 375-380.

19. Ferreira JS, Diettrich SHC, Pedro DA. Influência da prática de atividade física sobre a qualidade de vida de usuários do SUS. Saúde Debate 2015; 39 (106): 792-801.

\section{DADOS DOS AUTORES}

\section{Marcella Mayara Sousa Silva}

Graduada em Educação Física. Instituto Federal de Educação, Ciência e Tecnologia do Ceará (IFCE). Profissional de Educação Física. Canindé/CE - Brasil. marcellamayara2121@ gmail.com

\section{Francisco Laerton Teixeira Guerra}

Graduado em Educação Física. Instituto Federal de Educação, Ciência e Tecnologia do Ceará (IFCE). Profissional de Educação Física. Canindé/CE - Brasil. laertonguerra@gmail.com

\section{MARINA LiRA BATISTA}

Graduada em Educação Física. Instituto Federal de Educação, Ciência e Tecnologia do Ceará (IFCE). Profissional de Educação Física. Canindé/CE - Brasil. marinalira.20@gmail.com 


\section{Lidalvane Silva Lopes}

Graduada em Educação Física. Secretaria Municipal de Saúde de Canindé/CE. Profissional de Educação Física. Canindé/CE - Brasil. lidalvane@gmail.com

\section{Samara Moura Barreto de Abreu}

Mestre em Educação. Instituto Federal de Educação, Ciência e Tecnologia do Ceará (IFCE). Profissional de Educação Física. Fortaleza/CE - Brasil. samaraef@hotmail.com

\section{Nilson Vieira Pinto}

Doutor em Biotecnologia. Instituto Federal de Educação, Ciência e Tecnologia do Ceará (IFCE). Profissional de Educação Física. Fortaleza/CE - Brasil.nvp.ifce@gmail.com

Submetido em: 20-1-2019

Aceito em: 3-10-2019 